\title{
EMSAM (deprenyl patch): how a promising antidepressant was underutilized
}

This article was published in the following Dove Press journal:

Neuropsychiatric Disease and Treatment

6 October 2014

Number of times this article has been viewed

\author{
Gregory M Asnis ${ }^{1,2}$ \\ Margaret A Henderson² \\ 'Department of Psychiatry and \\ Behavioral Sciences, Albert Einstein \\ College of Medicine, New York, NY, \\ USA; ${ }^{2}$ Anxiety and Depression Clinic, \\ Montefiore Medical Center, New York, \\ NY, USA
}

\begin{abstract}
The EMSAM patch is a unique monoamine oxidase inhibitor (MAOI) being the only antidepressant utilizing a transdermal delivery system. This was welcomed by clinicians who hoped that EMSAM would be better tolerated than oral MAOIs and non-MAOI antidepressants, as well as being effective for treatment in a wide spectrum of depressed patients including atypical depression, bipolar depression, and refractory depression. Unfortunately, the clinical use of EMSAM has been underutilized and its potential usefulness overlooked. This article suggests that fear of possible side effects, particularly the "cheese reaction" and serotonin syndrome, are some of the main contributors to underutilization by clinicians. These risks have been significantly exaggerated with the $6 \mathrm{mg}$ /day dose not even requiring a special diet. Other contributing factors leading to underutilization are reviewed such as: the lack of studies addressing many important clinical questions; inadequate data analyses; not evaluating the effect of EMSAM on comorbid psychiatric conditions, particularly anxiety disorders; lack of antidepressant comparators versus EMSAM; no dose-response relationship examined; various depressive subtypes and conditions are unexplored, eg, bipolar depression and refractory depression; poor insurance coverage for an expensive medication; as well as minimal marketing efforts and postmarketing studies. On the other hand, many potential advantages of EMSAM are not highlighted enough in the literature and by pharmaceutical companies which might have increased clinical interest and utilization of the antidepressant. For example, the advantages of EMSAM include: avoidance of swallowing issues, as can be seen with oral antidepressants; minimal side effects, probably due to a favorable pharmacokinetic profile; minimal evidence of suicidal behavior, probably relating to the transdermal route of administration; low rates of inducing hypomanic/manic episodes; as well as significant efficacy in "anxious depression" and atypical depression. Recent efforts in conducting some post hoc analyses and presentations on EMSAM may yet stimulate further clinical interest and use of this antidepressant.
\end{abstract}

Keywords: EMSAM patch, MAOI, tyramine-free diet

\section{Introduction}

This article reviews how and why EMSAM, a novel monoamine oxidase (MAO) inhibitor (MAOI) antidepressant, has been significantly neglected and underused by the medical community despite being US Food and Drug Administration (FDA)approved for over 7 years. This approval was welcomed by academicians who believed that EMSAM would provide patients with the first antidepressant that had a new delivery system - a transdermal patch. This would minimize side effects and thus allow patients to more easily tolerate treatment with a highly effective class of antidepressants, the MAOIs.

In the last decade, researchers have demonstrated that although the pharmacotherapeutic treatment of major depressive disorder (MDD) was frequently effective, less than half of patients failed to have a remission of their illness whilst taking routine
Correspondence: Gregory M Asnis Montefiore Medical Center, III East 2 I Oth Street, Bronx, New York, 10467 NY, USA

Tel +I 9175979408

$\mathrm{Fax}+\mathrm{I} 7188824735$

Email asnisarts@aol.com 
antidepressants and, therefore, continued to suffer. This was most recently highlighted in the National Institute of Mental Health (NIMH)-sponsored STAR*D Study where only $47 \%$ of patients responded (an improvement of at least $50 \%$ from baseline) to treatment with citalopram, a selective serotonin re-uptake inhibitor (SSRI), and only about $30 \%$ had a remission of their illness. Furthermore, a relapse occurred in $40 \%$ of remitted patients when followed over 1 year's duration, despite continuing antidepressant treatment. ${ }^{1}$ These disappointing results for STAR*D and other antidepressant trials may be partially related to the inefficacy of antidepressants in many depressed patients but may also be related to patient noncompliance and medication side effects. Noncompliance is highly prevalent, occurring in as many as $68 \%$ of patients during the first 3 months of acute antidepressant treatment. ${ }^{2}$ In the STAR*D study, over $56 \%$ of citalopramtreated patients experienced significant side effects ${ }^{3}$ which highly predicted a poor treatment response. ${ }^{4}$ In an attempt to improve efficacy and compliance of antidepressants and decrease their side effects, new antidepressants with different mechanisms of action, modifications to their structure, and alterations in the delivery system have been sought. Liquid preparations of various antidepressants (eg, escitalopram, fluoxetine, doxepin) and a disintegrating form of an antidepressant tablet (Remeron Soltab) that dissolves within the mouth in 40 seconds have been utilized in populations to avoid swallowing problems and to facilitate compliance..$^{2,5-7}$ Fluoxetine has been developed as a once-a-week tablet to increase compliance from missed doses from daily administrations. ${ }^{6}$ Paroxetine CR (controlled release) was developed to decrease gastrointestinal side effects, particularly nausea, by being absorbed more in the duodenum than the stomach. ${ }^{6}$ Lastly, intravenous antidepressants (eg, clomipramine, citalopram, amitriptyline) have been developed (used in Europe) to potentially increase efficacy and possibly induce a faster antidepressant response since this route theoretically avoids first-pass metabolism and has favorable pharmacokinetics. ${ }^{8}$ These studies have failed to show any clinical advantages in efficacy for these altered forms of antidepressants and those with different routes of administration, although compliance may have improved. ${ }^{2,4-8}$

Recently (2008), EMSAM ([R]-[-]-N, 2-dimethyl-N2-propynylphenethylamine), a MAOI, was developed and approved as an alternative antidepressant with a novel transdermal delivery system. The transdermal delivery system was believed to offer a number of unique advantages. This mode of administration (transdermal) has provided for a beneficial pharmacokinetic profile, avoiding first-pass metabolism with gradual absorption over 24 hours and reduced absorption peaks. ${ }^{9}$ EMSAM, at low doses, is a selective MAO-B inhibitor (MAOI-B); at the doses approved (6-12 mg/ 24 hours), it is actually both a MAO-A inhibitor (MAOI-A) and $\mathrm{MAOI}-\mathrm{B}$. But since transdermal administration avoids direct gastrointestinal exposure and first-pass effects, there is no significant MAOI-A inhibition in the gut. A tyraminefree diet is unnecessary for the dose of $6 \mathrm{mg} / 24$ hours but is required for higher doses ( $9 \mathrm{mg}$ and $12 \mathrm{mg}$ dose) due to limited safety data for higher doses as judged by the FDA. ${ }^{10}$ The side effect profile for EMSAM $6 \mathrm{mg}$ was no different than placebo except for an increased incidence of patch-site reactions (mild contact dermatitis). ${ }^{10,11}$ In summary, EMSAM has been shown to be a highly effective antidepressant for MDD (for acute and maintenance treatment) in double-blind placebo-controlled studies with a reduced risk of many of the side effects noted for oral MAOIs, particularly the "cheese reaction". ${ }^{11-14}$ There have been three pivotal short-term (6-8 weeks), double-blind, placebo-controlled studies - two of these being a fixed dose of $6 \mathrm{mg}^{11,12}$ and one being a flexible dose study of 6-12 $\mathrm{mg}^{13}$ - and one long-term (52 weeks), double-blind, placebo-substitution fixed dose (6 mg) study. ${ }^{14}$ Interestingly, one of the short-term studies found drugplacebo differences starting as early as the end of week 1 of treatment, suggesting an early onset of action not usually seen with antidepressants. ${ }^{11}$

Thus, EMSAM is an alternative antidepressant that is highly effective and well-tolerated with a unique delivery system. The need for alternative antidepressants is obvious. This is highlighted by the STAR*D study finding that failing to respond to one antidepressant does not preclude responding to subsequent antidepressants. ${ }^{1,3}$ Nonetheless, whether EMSAM offers any clinical advantages with its unique transdermal delivery system awaits clarification.

The central question for our paper is why has EMSAM not been more widely prescribed by health professionals? This is even more bewildering since EMSAM is a MAOI, a class of drugs that may be one of the most effective of the antidepressants. Our review will also attempt to clarify the place of MAOIs, particularly EMSAM, in psychiatry.

MAOIs were first developed in the late 1950s for the treatment of MDD. Unfortunately, as a class of antidepressants, MAOIs have been misunderstood and underutilized. The discovery that MAOIs were effective in depression came by serendipity; a MAOI that was an effective antituberculosis drug, iproniazid, helped the depression of a patient with tuberculosis. Subsequent development and usage of other FDA-approved MAOIs for depression was accompanied 
with a number of patients having a hypertensive crisis, with some having strokes. These previously unrecognized adverse events led the FDA in 1964 to temporarily remove these drugs from the marketplace until the etiology of these adverse events could be clarified and precautions set up to prevent them. With the cheese reaction understood and the need for a tyramine-free diet (and also avoiding sympathomimetic drugs), MAOIs were reintroduced into psychiatry within a year after their discontinuation. ${ }^{15}$ Besides the need to restrict one's diet, avoiding certain foods and medicines which might induce a serotonin syndrome was also a mandate.

Despite these restrictions, MAOIs have been found to be more effective than tricyclic antidepressants in the treatment of atypical depression. ${ }^{16}$ Whether MAOIs offer any advantage over SSRIs for atypical depression still remains unclear. ${ }^{17}$ MAOIs are not only helpful for atypical depression but also are effective for non-atypical depression. McGrath et al found MAOIs were even effective in melancholia. ${ }^{18}$ Despite the efficacy of MAOIs in these conditions, clinicians generally recommend starting with SSRIs prior to considering a treatment course with an MAOI due to SSRIs' ease of use, no concerns for restrictive diets, and minimal worries about interactions with other medications. , $^{5,19}$

MAOIs have also been recommended as one of the treatment strategies in drug-refractory depression. ${ }^{6,20}$ As reviewed by McGrath et al, six studies (four double-blind and two open-label studies) assessed tranylcypromine, a MAOI, in refractory depression; response rates ranged from $29 \%$ to $75 \%$ with a median response rate of $50 \%{ }^{20}$ Large-scale studies directly addressing this topic have been lacking. Recently, the STAR*D project evaluated the efficacy of tranylcypromine, an irreversible MAOI, in an open-label study in patients with an MDD who were treatment refractory to a 12-14 weeks, course of citalopram (an SSRI), and two subsequent antidepressant treatment trials, for their refractory state. ${ }^{20}$ These refractory patients were randomized to receive either tranylcypromine $(n=58)$ or mirtazapine plus venlafaxine combination $(n=51)$. The remission rate for tranylcypromine was not significantly different from the comparator $(6.9 \%$ versus $13.7 \%$, respectively). Furthermore, the mean dose at the end of the study was less than $37 \mathrm{mg} /$ day, a dose which may have been subtherapeutic for many. Apparently, many of these refractory depressed patients were very sensitive to side effects of the MAOI, with $41 \%$ of MAOI-treated patients discontinuing the drug due to adverse events in comparison to $22 \%$ for the comparator group $(P<0.05)$. This sensitivity to adverse events in the MAOI group may also have led to the minimal dose received (patients could have been administered from 10 to $60 \mathrm{mg} /$ day) which may have been subtherapeutic for many. Interestingly, in a small study of depressed elderly patients $(n=16)$ who were refractory to at least two antidepressants, patients responded to $60 \mathrm{mg}$ /day of oral selegiline, the same drug making up the EMSAM patch. At this high oral dose, the selectivity of this compound for MAOI-B probably was lost. ${ }^{21}$ Furthermore, two recent case studies of refractory depression demonstrated a full response to EMSAM $6 \mathrm{mg} / 24$ hours. ${ }^{22,23}$ Thus, MAOIs, including EMSAM, may be helpful in refractory depression.

MAOIs may also be helpful in the treatment of bipolar depression (although not as researched as in unipolar depression) ${ }^{6,24}$ Bipolar depression is a difficult mood disorder to treat with a large literature suggesting that antidepressants can induce cycling, create episode instability, and contribute to an unsustained recovery. ${ }^{6,24,25}$. Thus, MAOIs may be one of the least risky in this regard and be superior in efficacy to SSRIs.

Thus, MAOIs appear to be an alternative antidepressant treatment, and may be particularly effective in refractory depression, atypical depression, melancholia, and possibly bipolar depression. Unfortunately, side effects and dietary issues may limit its general usefulness. Recently, selegiline, a MAOI-B, was developed as a transdermal patch (EMSAM) in an attempt to develop a more user-friendly MAOI. The availability of a new MAOI, EMSAM, that is an easily applied transdermal antidepressant preparation should provide an antidepressant that is easier to administer and more tolerable than oral MAOI preparations. This is clinically relevant since the use of oral MAOIs is clearly limited by their potential side effects (as seen in the STAR*D project, oral MAOIs are not well-tolerated by a significant subgroup), leading to limitation of dosage and/or early discontinuation..$^{20}$

\section{Reluctance in prescribing MAOls}

As part of understanding the place and use of EMSAM in psychiatry, one must understand the general use of MAOIs in psychiatry. There clearly is great reluctance amongst clinicians in prescribing MAOIs. In two surveys given anonymously, $75 \%-83 \%$ of practicing psychiatrists prescribed MAOIs occasionally or not at all. This under-use appears to be worsening over time with $25 \%$ of psychiatrists reporting frequently using MAOIs in a 1990 study versus $2 \%$ in a 1999 study. Interestingly, about 39\% of psychiatrists had not prescribed a MAOI in the prior 3 years. ${ }^{26,27}$ A recently published article assessing antidepressant usage in the elderly found that irreversible MAOIs were rarely prescribed. ${ }^{28}$ The review tracked a decade of new prescriptions of irreversible 
MAOIs from 1997 to 2007 and found that usage was steadily decreasing from 3.1/100,000 in 1997 to 1.4/100,000 in 2007. The dwindling and limited usage of MAOIs is highlighted by the group's finding that, of those elderly that were treated with antidepressants, less than $0.2 \%$ were treated with MAOIs. ${ }^{28}$ The surprising limited usage of MAOIs is particularly striking since the American Psychiatric Association, the British Association of Psychopharmacology, and the Canadian Network for Mood and Anxiety Treatments guidelines have recommended MAOIs as a second- or third-line option for the treatment of atypical depression, refractory depression, and bipolar depression. 5,6,29,30

Regarding the usage of transdermal EMSAM, a recent report found that after 1 year post-FDA approval, 30\% of MAOI prescriptions for depression were for EMSAM. But most disappointingly, only $0.1 \%$ of antidepressant prescriptions were for EMSAM. ${ }^{31}$ Thus, marketing of EMSAM was successful in terms of it being one of the most prevalent MAOIs prescribed; nonetheless, it rarely was prescribed among antidepressants. Thus, at this point in time, MAOIs are rarely utilized in the treatment of depression. Nonetheless, why would there be a hesitancy in prescribing EMSAM, a new MAOI with apparent significant safety over previous oral MAOIs?

What follows is a discussion of possible factors that may have contributed to the underutilization of EMSAM. Of course, these suggestions are speculative, based on a literature review, and years of supervising and teaching psychiatric residents, fellows, and attending physicians. Unfortunately, there has not been a survey focusing on the hesitance of use of EMSAM by psychiatrists.

\section{Fear of possible side effects and shortcomings in EMSAM development The cheese reaction}

The first area that probably contributed most widely to the hesitancy of prescribing EMSAM by clinicians is a continued fear of an MAOI-induced hypertensive crisis (the tyraminebased cheese reaction), most notably seen in the 1960 s prior to dietary restrictions being imposed, as well as drug-drug interactions and serotonin syndrome. Prior to realizing the need to avoid tyramine-rich diets, significant hypertension was estimated to occur in $2.4 \%-25 \%$ of cases taking oral MAOIs ${ }^{32}$ with up to $25 \%$ of these patients developing a stroke or even dying; ${ }^{33}$ over 40 cases of tyramine-induced hypertensive crisis were reported. ${ }^{34,35}$ During the 1-year withdrawal of MAOIs from the marketplace, studies demonstrated that the tyramine cheese reaction and a number of drug-drug interactions were responsible for the adverse reactions. After the reintroduction of MAOIs back into the marketplace in 1965 with various precautions duly noted, many clinicians and patients have been hesitant about using MAOIs due to the need to carefully follow such restrictions and the relative ease of use of other antidepressants, particularly the SSRIs. ${ }^{36}$

Thus, the fear of a cheese reaction may still linger regarding prescribing EMSAM. In fact, the literature suggests that a cheese reaction secondary to EMSAM is a rare event. In the clinical trial database of 2,036 subjects, no subject had an acute hypertensive reaction to EMSAM. ${ }^{10}$ This is remarkable in that most of the trials did not follow a tyramine-free diet. In the EMSAM clinical trials, 357 patients received 9 or $12 \mathrm{mg} /$ day, doses that the FDA have now required to be followed with a tyramine-free diet. ${ }^{10}$ Since doses greater than $6 \mathrm{mg}$ ( 9 and $12 \mathrm{mg}$ ) had a significantly increased tyraminepressor response (considerably less than oral MAOIs but safe considering the tyramine content of a tyramine-rich diet) and that there was insufficient tyramine-pressor response data for the 9 and $12 \mathrm{mg}$ /day doses, the FDA mandated a tyramine-free diet for these two higher doses. ${ }^{10}$ A postmarketing analysis recently reported that of 29,141 patients treated with EMSAM, 13 cases had a hypertensive crisis. Of these 13 cases, eight were subsequently deemed not to have a hypertensive reaction after careful review. Furthermore, of the five confirmed cases, only two were determined to be secondary to a true tyramine reaction and three were associated with another drug the patient was taking. ${ }^{37}$

Thus, at least with EMSAM, the risk of a cheese reaction appears to be minimal. Nonetheless, due to the possibility of a cheese reaction to 9 and $12 \mathrm{mg} /$ day doses, a tyramine-free diet must be followed as mandated by the FDA.

Clinicians and patients often feel that the MAOI diet is highly restrictive and onerous, making it hard to follow and not foolproof. In fact, it is one of the major factors described in the limited use of oral MAOIs ${ }^{27}$ and probably is an important deterrent to prescribing EMSAM .

The tyramine-free diet has been simplified and streamlined, becoming more user-friendly for patients. Sharing the extent of this safety record may not have been significantly stressed in the literature or by pharmaceutical companies. Since the FDA does require that tyramine-free diets be followed for EMSAM $9 \mathrm{mg}$ and $12 \mathrm{mg}$ doses, it is imperative for clinicians and patients to have a full understanding of this diet, the potential risks in not following it, how restrictive it is, etc. Education for the physician and the patient regarding tyramine-free diets has been lacking. 
Tyramine, an indirect sympathomimetic agent, is present in various foods and beverages. It is normally metabolized in the gut by MAO-A enzyme. When it is not metabolized due to MAO inhibition, it enters the circulation and is taken up by noradrenergic neurons, displacing norepinephrine. The latter is extruded from the neuron and contributes to a potential hypertensive crisis with the risk of stroke and possible death. ${ }^{36}$ Thus, it is not surprising that clinicians are reluctant to prescribe MAOIs and patients are reluctant to take MAOIs. Tyraminefree diets, although originally limited to a few aged cheeses, became extended to over 70 foods. It appears that the extent of these restrictions have been unnecessary. A carefully detailed analysis of the tyramine content of these various foods/beverages by Shulman et al (1996) ${ }^{38}$ found that only a limited number had dangerously high tyramine content (greater than or equal to $6 \mathrm{mg} /$ serving) including chicken liver aged 9 days (63.84 mg/30 g), air-dried sausage (7.56 g/30 g), soy sauce $(0.941 \mathrm{mg} / \mathrm{mL})$, and sauerkraut $(7.75 \mathrm{mg} / 250 \mathrm{~g})$. Many of the foods that were previously thought to be dangerous have negligible tyramine content. Even amongst aged cheeses, mozzarella cheese has minimal tyramine content. In addition, most common cheeses such as cottage cheese, cream cheese, or ricotta have low tyramine content. Furthermore, chianti wine and canned beers have relatively low levels (although tap beer has higher tyramine content and should be avoided). The authors recommended that only those foods with high tyramine content per serving should be absolutely restricted with the remainder being "safe or safe in moderation". ${ }^{38}$ Such an understanding puts this potentially foreboding risk in perspective and helps remove the absolute fear that resides in clinicians and patients regarding MAOIs.

Although MAOI diets may be one factor limiting the use of EMSAM, it cannot be the major factor; this is highlighted by the fact that there are no dietary restrictions for the $6 \mathrm{mg}$ dose (an effective antidepressant dose) and it still is underprescribed.

\section{Serotonin syndrome}

Drug interactions with MAOIs have been another public health concern. Serotonin-promoting drugs can lead to a "serotonin syndrome", a state of excess of serotonin. A triad of clinical symptoms occur in this disorder, consisting of an altered mental state, neuromuscular hyperactivity, and autonomic hyperactivity. Symptoms can include hyperthermia, rigidity, myoclonus, delirium, and potentially death. ${ }^{39}$ Coadministered drugs that are most commonly implicated in serotonin syndrome are antidepressants that inhibit serotonin reuptake such as tricyclic antidepressants and SSRIs, psychostimulants such as amphetamine, and serotonin-promoting analgesics and opiates such as tramadol, meperidine, and methadone. ${ }^{39}$ The tragic case of Libby Zion of a drug-drug interaction with MAOIs was highly publicized in 1984 and subsequently for years with wide press coverage; she was administered one dose of an opiate, meperidine, by a medical intern at a university hospital while she was being treated with an MAOI, phenelzine (there were also traces of cocaine, a sympathomimetic, in her system). Her administering physician was unaware of the potential lethal interactions. The patient developed a serotonin syndrome and died within hours. ${ }^{40}$ This widely publicized case is not only an example of a disastrous drug-drug interaction, but the memory of this case is still with many and may still be a deterrent for clinicians in prescribing MAOIs. Thus, physicians must be careful for drug-drug interactions in patients concurrently being treated with MAOIs. This potential interaction can occur for EMSAM even at the lowest approved dose, $6 \mathrm{mg} /$ day.

Nonetheless, particular interactions of EMSAM with serotonin-promoting drugs have been scarce. Patients treated with EMSAM have been cotreated with SSRIs without consequences. ${ }^{10}$ Recently, a case report found that a patient treated with EMSAM could periodically take over-the-counter sympathomimetic agents such as cold remedies and weightloss formulations without harm. ${ }^{41}$ In evaluating the EMSAM clinical trial data, Robinson and Amsterdam reported that over 100 subjects had taken a prohibited medication, particularly oral decongestants, antitussives, and various opioids. Interestingly, only one of these subject developed an adverse event, premature ventricular contractions, which occurred after taking pseudoephedrine. The premature ventricular contractions dissipated after discontinuing pseudoephedrine. ${ }^{10}$

In addition, of all the cases in the database, only one had developed a validated serotonin syndrome. The patient in this case was secretly taking a number of contraindicated medications - nortriptyline, bupropion, and a sympathomimetic drug for weight control - as well as using double the amount of prescribed EMSAM - two $12 \mathrm{mg}$ patches - at the same time. This patient needed to be intubated but responded to supportive care with full recovery. ${ }^{10}$

Thus, despite some precautionary measures when using EMSAM, it appears to be relatively safe. Except for a few isolated foods that should be absolutely restricted with higher doses of EMSAM ( 9 and $12 \mathrm{mg}$ /day), the diet is not an important issue. Regarding drug-drug interactions, particularly serotonin syndrome, this is apparently very rare. Nonetheless, due to the life-threatening seriousness of this 
syndrome, this prohibition must be respected. This does potentially limit the usefulness of EMSAM in patient groups that require significant analgesics or need sympathomimetics such as asthmatics, patients with attention deficit disorders, or in patients prone to use opiates, particularly those with a history of drug abuse.

\section{Paucity of studies looking at comorbid psychiatric conditions}

MDD is frequently comorbid with other psychiatric diagnoses, especially anxiety disorders at a rate of approximately $60 \% .^{42}$ Therefore, clinicians would like to know whether a specific antidepressant is helpful not only in the treatment of major depression but also for other a comorbid illnesses. In fact, most newer-generation antidepressants, particularly SSRIs and serotonin-norepinephrine reuptake inhibitors, are effective and FDA-approved for most anxiety disorders such as panic disorder, generalized anxiety disorder, obsessivecompulsive disorder, and post-traumatic stress disorder. ${ }^{43}$

How effective is EMSAM in treating comorbid anxiety disorders in MDD? One of the failures in the development of EMSAM was not evaluating this drug in anxiety disorders, either primary or secondary to MDD. Even where an anxiety disorder was secondary to MDD as was permitted in three of the four published double-blind clinical trials, ${ }^{11,12,14}$ the effect of EMSAM on the anxiety disorder was not presented. Even the actual comorbid anxiety disorders included and the number of cases in each disorder were not described in the reports. An important analysis may have been missed since earlier literature on MAOIs suggested that they were very effective in anxiety disorders. ${ }^{43}$ It is also remotely possible that EMSAM may not only be ineffective for anxiety disorders but might exacerbate them. For example, fluoxetine, an SSRI antidepressant, has been reported to induce anxiety (jitteriness syndrome) in a subset of patients, particularly those who have anxiety disorders. ${ }^{44}$ Clearly, knowing what effect EMSAM has on comorbid anxiety disorders (a post hoc analysis on the clinical trial data would help answer this important clinical question) is of clinical relevance. Thus, a post hoc analysis awaits.

\section{Lack of studies with antidepressant comparators}

None of the registration studies evaluating EMSAM in MDD used an antidepressant comparator; the FDA approval process does not mandate an antidepressant comparator (it does mandate a placebo comparator). Clinicians surely would have felt more comfortable knowing how EMSAM compared to another antidepressant (particularly an FDA-approved antidepressant) regarding clinical efficacy and side effects, even if such a study was not powered to demonstrate true superiority of one antidepressant over another. The ultimate question of whether EMSAM offers any advantage clinically over other antidepressants for MDD remains unanswered. In general, there is a scarcity of antidepressant-comparator studies. A recent meta-analysis conducted by Cipriani et al evaluating 12 new-generation antidepressants found that four antidepressants (mirtazapine, escitalopram, venlafaxine, and sertraline) were more effective than four others (duloxetine, fluoxetine, paroxetine, and reboxetine). ${ }^{45}$ As reviewed by Stahl, meta-analyses like this one have certain limitations such as dosing differences across antidepressants, etc. ${ }^{46}$ A well-designed study comparing many currently approved antidepressants including EMSAM in the treatment of MDD would be welcomed. Such a study would have to be powered adequately and therefore be large. It would probably have to be supported by NIMH, similar to the recent STAR*D project. $^{1,3}$ The latter compared a number of antidepressants (and combinations) to one another and was powered to detect potential differences in refractory MDD. If EMSAM proved to be one of the more effective antidepressants assessed, clinicians no doubt would be more interested and willing to consider using EMSAM in MDD.

\section{High cost of medication/insurance coverage}

The EMSAM patch (6 mg, $9 \mathrm{mg}$, or $12 \mathrm{mg}$ ) costs approximately $\$ 20$ per patch or over $\$ 600 /$ month for treatment. ${ }^{47}$ This high cost is frequently not covered by insurance and managedcare companies. In contrast, other antidepressants, including SSRIs, cost between \$7 and \$154 per month depending on generic or brand designations. ${ }^{48}$ Regarding insurance coverage, if EMSAM is covered, patients frequently must first fail to respond to one or two other antidepressants and also be responsible for larger copayments. Thus, for many patients, treatment with EMSAM patch is significantly costly and thus may be prohibitive. This may change when EMSAM becomes generic in the future.

\section{A dose-response relationship was never established}

The effective dose range of EMSAM in the treatment of MDD is from 6 to $12 \mathrm{mg} /$ day. Three of the double-blind placebocontrolled studies focused exclusively on $6 \mathrm{mg} /$ day. ${ }^{11,12,14}$ Only one of the double-blind placebo-controlled acute studies ( 8 weeks' study) used a flexible dose starting at $6 \mathrm{mg}$ and raising 
the dose by $3 \mathrm{mg} /$ day every 2 weeks (at the discretion of the investigator) to a maximum of $12 \mathrm{mg} /$ day. ${ }^{13}$ The majority of the patients had their dose raised to $9 \mathrm{mg}$ with about $50 \%$ reaching the $12 \mathrm{mg}$ dose (60-70 patients). In the one longterm study (62 weeks), only the dose of $6 \mathrm{mg}$ was assessed. ${ }^{14}$ Thus, only a few patients were studied at the higher two doses - $9 \mathrm{mg}$ and $12 \mathrm{mg} /$ day - and only during the acute phase of treatment. In summary, a study would have to compare various fixed doses of EMSAM (6 mg versus $9 \mathrm{mg}$ versus $12 \mathrm{mg} / 24$ hours) versus placebo to truly answer whether a dose-response relationship exists. Thus, the clinician cannot be assured that raising the dose beyond $6 \mathrm{mg} /$ day offers an increased likelihood of having a clinical response. The clinician can be assured that raising the dose increases the likelihood of side effects and mandates the use of a tyramine-free diet.

\section{A limited age range was studied}

MDD affects a wide age range from childhood to the elderly. Antidepressants have been predominantly studied and FDAapproved for the adult population: $18-65$ years of age. There has been a paucity of antidepressant registration studies for children and adolescents as well as the geriatric population. At this point in time, only fluoxetine is approved for children aged 8-12 years; both fluoxetine and escitalopram are approved for adolescents aged 12-17 years, and fluoxetine for the geriatric population. ${ }^{49,50}$ Regarding antidepressants in MDD in childhood and adolescents, there appears to be a large placebo response with rates averaging about $48 \% .^{51}$ Furthermore, antidepressants appear to be less effective and take longer to work in older-adult depressives (greater than 65 years old) than younger-adult depressives; in addition, older depressives are more likely to experience a relapse during antidepressant treatment. ${ }^{6,52}$ Lastly, children, adolescents, and the elderly appear to be particularly sensitive to antidepressant-induced side effects. ${ }^{50,53}$

Thus, a new antidepressant such as EMSAM that might be particularly effective and well-tolerated in the young and elderly would be of special interest to clinicians. Interestingly, MAOIs theoretically, may be particularly effective in the elderly since brain-MAO activity increases as one ages ${ }^{54}$ and MAO levels in the brains of depressed patients are elevated, ${ }^{55}$ both conditions possibly being additive and contributing to lowering monoamine levels in geriatric depression. In addition, patients with geriatric depression appear to have serotonin reuptake transporters that are resistant to inhibition by antidepressants, the main mechanism of action of many antidepressants, particularly the SSRIs. ${ }^{56}$ In general, there has been a scarcity of studies of MAOIs in the elderly.
EMSAM clinical trials (controlled and uncontrolled) have included depressed patients 65 years and older (up to 95 years old) in as many as 198 patients (198 of 2,036 clinical trial patients). ${ }^{10}$ Unfortunately, there were no analyses as to whether aging had a significant effect on the efficacy and safety of EMSAM. This kind of analysis was possible but not performed in two of the four pivotal controlled and published trials which included patients 65 years and older. ${ }^{13,14}$

Regarding the possible efficacy and safety of EMSAM in the treatment of childhood and adolescent depression, a recent Phase $1 \mathrm{~V}$ postmarketing placebo-controlled study (flexible dose, 6-12 mg/day) was just completed in 300 adolescents ages 12-17 years. EMSAM was well-tolerated but there was no significant drug-placebo response; the placebo response rate was over 59\%, making drug superiority next to impossible. ${ }^{57}$ EMSAM has not been studied in childhood depression. Clinicians might have found it useful to know EMSAM's effect across all age ranges, particularly the elderly. If it was clearly demonstrated to be safe and effective in these groups, this information might have encouraged a wider use of EMSAM.

\section{Minimal clinical trials, minimal marketing force, and minimal postmarketing studies} The pharmaceutical company that developed EMSAM (Somerset Pharmaceuticals, Inc., Tampa, FL, USA) was relatively small. Therefore, the breadth of clinical trials and postmarketing studies were probably restricted due to limited financial resources. Furthermore, the marketing team was also probably too small for the challenge of getting clinicians who were, by history, hesitant to use MAOIs to try a new MAOI, EMSAM. For a limited period in 2006, Bristol-Myers Squibb (New York, NY, USA) was licensed to market EMSAM, which was rather unsuccessful. This may have been due to competing resources within the company in marketing their own highly successful psychotropic medication, aripiprazole. In the last few years, Dey Pharma (Basking Ridge, NJ, USA) and now Mylan Pharmaceuticals (Morgantown, WV, USA) took over the marketing of EMSAM with the hope of increasing clinical usage. Whether these small companies can succeed is unclear.

\section{Many depression subtypes not explored}

A number of subtypes and/or varieties of MDD can have significant bearing as to whether an antidepressant is effective. For example, psychotic depression responds poorly to antidepressants alone compared to antidepressants plus antipsychotics or electroconvulsive therapy. ${ }^{58}$ As described 
earlier, a literature exists that oral MAOIs appear to be particularly effective in atypical depression; ${ }^{16,17}$ in addition, oral MAOIs, in contrast to other antidepressants, are effective in bipolar depression, ${ }^{6,24}$ and most importantly, refractory depression. ${ }^{6,20}$ Unfortunately, EMSAM was not specifically evaluated for these depressive subtypes in the original clinical trials and reports (a recent post hoc analysis on atypical depression will be presented in the section "Equally effective in atypical and non-atypical depression"). Regarding refractory depression and bipolar depression subtypes, EMSAM clinical trials excluded depressed patients with these subtypes. Two case studies did find that EMSAM $6 \mathrm{mg}$ was effective in two patients with refractory depression. ${ }^{22,23}$ Thus, if EMSAM's effectiveness in these subtypes could be demonstrated, it would encourage its clinical use.

\section{Other treatment options are widely available}

A multitude of various antidepressants from various drug classes have been approved and are used as a primary treatment or when someone is refractory to a particular antidepressant in MDD. ${ }^{5,6}$ Most of these antidepressants are more user-friendly (no need to follow a diet, etc) than EMSAM. The antidepressant marketplace is highly competitive and difficult for a new antidepressant like EMSAM to gain market share. Regarding refractory depression, a number of treatment options have become popular clinically over the years - some FDA-approved (eg, adjunctive use of atypical antipsychotics) and many not FDA-approved (potentiating an antidepressant with lithium, thyroid, etc). ${ }^{5,6}$ EMSAM was reported to be effective in refractory depression in only two case studies. $^{22,23}$

\section{Failure to point out potential advantages of EMSAM over other antidepressants No swallowing issues}

Many patients have problems swallowing oral medications. Their psychological and/or physical make-up leads to avoidance of swallowing any medications. This is particularly significant in children and adolescents ${ }^{59}$ as well as the elderly. ${ }^{60}$

\section{Decreased gastrointestinal sensitivity}

Many patients have clear gastrointestinal sensitivity to antidepressants, particularly SSRIs. These side effects, especially nausea and vomiting, frequently lead to noncompliance and or discontinuation of treatment. ${ }^{5,6}$ Interestingly, EMSAM has minimal gastrointestinal side effects (not significantly different from placebo) at its prescribed doses ( $6 \mathrm{mg} / \mathrm{day}, 9 \mathrm{mg} /$ day, and $12 \mathrm{mg} /$ day), ${ }^{10}$ which probably is related to its transdermal route of administration. This finding should make EMSAM user-friendly to patients and encourage clinical use.

\section{Better pharmacokinetic profile}

Another potential benefit of transdermal over oral administration of antidepressants is that there is a large intraindividual variability of absorption and metabolism of oral antidepressants in contrast to transdermal preparations, as demonstrated for selegiline. ${ }^{9}$ Marked variability with oral antidepressants has been known for some time; blood levels of individuals taking nortriptyline can vary by over tenfold ${ }^{61}$ In addition, the transdermal preparation provided a high and sustained concentration over the period of study with a bioavailability of $73 \%$, in contrast to the oral preparation which produced a rapid peak falling off in 1 hour of ingestion with a bioavailability of 4\%. ${ }^{9}$ Thus, EMSAM's better pharmacokinetic profile may contribute to it being well-tolerated.

\section{Decreased general adverse events}

Side effects or adverse events are frequent occurrences with antidepressant medication, and are the main cause of noncompliance and/or discontinuation of these medications. Although second-generation antidepressants such as SSRIs and serotonin-norepinephrine reuptake inhibitors are better tolerated than first-generation antidepressants like tricyclic antidepressants and oral MAOIs, they still induce a number of undesirable side effects. In stark comparison, EMSAM has very few side effects. At $6 \mathrm{mg}$, the lowest effective dose, EMSAM (in comparison to placebo) is not associated with an increased incidence of sexual dysfunction or gastrointestinal effects, two side effects that are troublesome with most antidepressants. The only side effect clearly associated with the $6 \mathrm{mg}$ patch was skin irritations which is commonly seen in transdermal medication applications $(22 \%) .{ }^{11}$ This specific side effect appears to be an interaction of medication plus skin patch since the placebo skin patch had a significantly lower rate $(12 \%)$ of irritation than the EMSAM patch. This dermatological side effect was usually of mild severity in the EMSAM clinical trials, rarely leading to discontinuing of medication. ${ }^{10}$ Even at higher doses of selegiline $(9 \mathrm{mg}$ and $12 \mathrm{mg}$ ), side effects, including insomnia, dry mouth, dizziness, nervousness, and abnormal dreams, were less than most antidepressants but greater than the $6 \mathrm{mg}$ EMSAM 
dose. The finding of only mild to moderate side effects for EMSAM, particularly for the $6 \mathrm{mg}$ dose, would have been better-highlighted if studies actually compared EMSAM to other antidepressants where many patients are not able to tolerate their side effects. EMSAM may be particularly useful for these patients. The reduced side effects noted for EMSAM may be due to the beneficial pharmacokinetic profile that a transdermal patch insures. The EMSAM patch was associated with lower peak concentration and slower times to reach the maximum serum concentration than the oral preparation. ${ }^{9}$

These minimal side effects are particularly striking when one compares EMSAM (a transdermal MAOI) to oral MAOIs. The latter not infrequently induces hypotension, edema, drowsiness, nausea, and other bothersome side effects which are rarely seen with EMSAM.,6,10

\section{Possibly reduced suicidal behavior}

Patients with MDD frequently exhibit suicidal behavior. A prior survey by our group found that over $25 \%$ of depressed outpatients had made prior suicide attempts, with over half having made multiple attempts in their lifetime while another $25 \%$ had suicidal ideation. ${ }^{62}$ Most of these suicide attempts were impulsive and involved overdoses with antidepressants and other medications. Although EMSAM has been FDA-approved since 2006, there have been a minimal number of reported cases of suicide attempts or completed suicides with this drug in the literature. In the premarketing clinical trial data set of 2,064 patients, there were no completed suicides. The package insert states that $0.5 \%(n=11)$ of EMSAM group versus $0.4 \%(n=3)$ of the placebo group had a suicidal event, violent behavior, or attempted overdose. ${ }^{63}$ Recently, Pae et al $(2012)^{37}$ presented a summary of postmarketing data on 29,141 patients that have been prescribed EMSAM. This updated report found that four cases had completed suicide and four more made suicide attempts. Of the completed suicides, all cases used multiple drugs, particularly those that were contraindicated with EMSAM usage, such as crystal methamphetamine and bupropion (taken in overdose quantities). One case did make an overdose with 28 EMSAM patches applied to him concomitantly with numerous doses of amphetamine, but survived. ${ }^{37}$ It would appear that the use of transdermal patches makes it more difficult to successfully overdose and, therefore, may protect someone from such impulsive attempts. Theoretically, it would be possible to administer enough EMSAM patches to successfully commit suicide although the lethal amount of patches necessary is unclear.
Although clearly rare events, a few isolated reports in the literature cite suicidal behavior with other transdermal patches. Wiesbrock et al and LoVecchio and Ramos each reported a successful suicide with fentanyl (an opioid) transdermal patches, with one patient applying 20 patches and the other eleven patches, respectively. ${ }^{64,65}$ Raber et al reported an unsuccessful suicide attempt with a patient ingesting a clonidine transdermal patch. ${ }^{65}$ Lastly, nine cases unsuccessfully tried to commit suicide with nicotine patches (two to 20 patches). ${ }^{66}$ Thus, the literature suggests that suicidal behavior rarely occurs with the use of transdermal patches. Therefore, EMSAM might be a preferred treatment in depressed patients who have a history of previous suicidal behavior or who are currently suicidal. Nonetheless, since there are no comparative studies comparing EMSAM to other antidepressants, this possible advantage for EMSAM awaits validation.

\section{Decreased risk of inducing a hypomanic/ manic episode}

All antidepressant treatments (including pharmacological treatments and nonpharmacological treatments such as electroconvulsive therapy and light therapy) have the potential of inducing a hypomanic/manic episode during treatment. ${ }^{6}$ Whether these troublesome adverse event are due to a toxic event or are related to an underlying bipolar disorder being present, either misdiagnosed as a unipolar depression or present in a subthreshold form of bipolar disorder, is unclear. ${ }^{25,68}$ Interestingly, a recent meta-analysis by Tondo et al found that MAOIs were the class of antidepressants with the lowest rate of hypomanic/manic induction (4.6\%) as compared to others such as SSRIs (8.7\%) and tricyclic antidepressants (TCAs) (12.7\%). ${ }^{69}$ Therefore, it is not surprising that EMSAM appears to have a relatively low risk of inducing a hypomanic/manic episode during treatment. An early analysis of Phase III clinical trial data found that $0.4 \%$ (8 of 2,036 patients) developed a hypomanic/manic episode. ${ }^{63}$ When evaluating only Phase III acute treatment clinical data that was placebo-controlled (five short term studies, 6-8 weeks' duration), two of 817 (0.24\%) EMSAM patients versus one of $668(0.15 \%)$ placebo patients had a hypomanic/ manic reaction (KB Portland, Medical Director of Dey Specialty, L.P., personal communication, December, 2012). In a 10-week open-label EMSAM study (where responders were entered into a double-blind placebo-controlled discontinuation phase lasting 52 weeks), the data are as follows: of 674 patients treated openly, one $(0.15 \%)$ had a hypomanic/ manic reaction; of the 321 patients responding to this 
10-week open phase and entering the 52-week double-blind discontinuation phase, two of $158(1.26 \%)$ patients continued on EMSAM had a manic reaction versus 0 of $163(0 \%)$ patients who were switched to placebo (KB Portland, Medical Director of Dey Specialty, L.P., personal communication, December, 2012). A recent analysis of postmarketing EMSAM data found that only $0.05 \%$ (16 of 29,141) patients had such a hypomanic/manic episode. ${ }^{37}$ The lower rates of manic/hypomanic episodes post-trial possibly relates to less structured evaluations (lack of clinical rating scales) for this adverse event. In summary, evaluating both clinical trial and postmarketing data, EMSAM rarely has been found to induce a hypomanic/manic reaction. This information should diminish any safety concerns and increase the interest of clinicians in prescribing EMSAM.

\section{Equally effective for anxious and non-anxious depression}

Anxiety is a frequent comorbid symptom in patients with MDD; approximately 53\% of MDD patients have significant anxiety symptoms, so called "anxious depression". ${ }^{70}$ Unfortunately, the presence of anxious depression in MDD is a negative predictor of antidepressant responsiveness. ${ }^{70,71}$ Nonetheless, some antidepressants appear to be more effective than others for anxious depression; for example, SSRIs were found to be more effective than bupropion. ${ }^{71}$ Therefore, clinicians would like to know whether a specific antidepressant is helpful not only in the treatment of MDD but also if marked anxiety or anxious depression is present.

The EMSAM clinical trials originally failed to assess the effect of EMSAM on anxiety symptoms or specifically anxious depression. This is unfortunate since earlier literature on oral MAOIs suggested that they were effective for various anxiety symptoms. ${ }^{72}$ Recently, Robinson and Portland ${ }^{73}$ presented a post hoc meta-analysis of the EMSAM clinical trial data (6-12 mg/24 hours) of three randomized, doubleblind, placebo-controlled studies dividing MDDs into anxious and non-anxious depression. They found that both anxious and non-anxious depressives did significantly better with EMSAM versus placebo. In addition, the clinical response of anxious depressives to EMSAM did not differ from that of non-anxious depressives. ${ }^{73}$ Communication of these post hoc results to clinicians is of importance and probably would increase interest in prescribing EMSAM to patients with MDD, particularly those with anxious depression. Whether EMSAM might be more effective than other antidepressants in anxious depression awaits to be studied.

\section{Equally effective in atypical and non-atypical depression}

Atypical depression represents approximately 19\%-29\% of patients with MDD. ${ }^{74,75}$ This sizable subgroup of depressed patients as defined by Asnis et al has a reactive mood with at least two of the following: hypersomnia, hyperphagia, leaden paralysis, or rejection sensitivity. ${ }^{74}$ Oral MAOIs have consistently been found be to more efficacious than placebo and TCAs in atypical depression. ${ }^{16,17}$ Other studies have been inconclusive as to whether SSRIs were significantly different than MAOIs in atypical depression. ${ }^{17}$ In contrast, The STAR*D study recently found that atypical depressives do not respond as well as non-atypical depressives to an SSRI, citalopram; atypical depressives had a lower remission rate to a course of citalopram treatment. Furthermore, those that remitted took longer to go into remission than non-atypicals. ${ }^{75}$

Unfortunately, the original clinical trials of EMSAM in MDD failed to address its clinical efficacy in atypical depression. Recently, Pae et al performed a post hoc analysis of five short-term placebo-controlled EMSAM trials. EMSAM was equally efficacious in atypical and non-atypical depressives. ${ }^{76}$ Whether EMSAM is more effective than non-MAOI antidepressants for atypical depression should be assessed. Nonetheless, these recent findings should provide clinicians at least a sense of confidence in administering EMSAM to patients with MDD since atypical depression did not have a negative influence on treatment response.

\section{Conclusion}

EMSAM is clearly an underutilized antidepressant which may be due partially to fears about MAOI side effects, clinical trials that did not address enough clinical questions, inadequate analyses of data, minimal postmarketing studies, and inadequate marketing in general. The pharmaceutical industry should not be shortsighted when developing a novel drug for the treatment of a highly prevalent disorder such as MDD. This is particularly relevant when the drug is an MAOI with a long prior history of adverse events (particularly the cheese reaction) leading to a number of deaths, with the FDA temporarily removing the drug from the market. The hesitancy to prescribe such drugs by clinicians is thus understandable, particularly when the marketplace has many other safer and less-restrictive antidepressant choices; therefore, the safety and potential efficacy of a new MAOI, even one that is touted to have many advantages over prior MAOIs and other antidepressants, must be demonstrated beyond any doubt. In an era of many antidepressant-treatment choices, 
why to prescribe a particular antidepressant must be clearly spelled out to ensure its usage. If misuse of a drug or idiosyncratic reactions to it could lead to medical mishaps, the clinical usage of it will be minimal. This is most relevant in the current harsh medical-legal environment where frequent public advertisements are aired soliciting patients to report potential harmful effects experienced from a drug so that litigation can proceed against a physician and pharmaceutical company.

As suggested in this article, a number of opportunities in researching and marketing EMSAM have been missed. Better education of the clinicians about MAOIs, particularly EMSAM, and more research assessing their uniqueness was and still is in order. Although EMSAM appears to have certain advantages over many antidepressants as outlined in this manuscript, the pharmaceutical company is limited in promoting some of them since they are not included in the drug's labeling. Recently, Dye and now Mylan Pharmaceutical took over the marketing of EMSAM from Bristol-Myers Squibb. With over 14 years left in the patent of EMSAM, Mylan has the opportunity to still conduct some further basic studies in MDD and provide important medical education to the health community. Both Dye and Mylan's recent attempts at reanalysis of some EMSAM data is promising and hopefully will provide a stimulus to clinicians to think of prescribing EMSAM more often in the treatment of MDD. Ultimately, a study comparing EMSAM to other antidepressants in MDD focusing on efficacy and tolerance would be clinically important. If EMSAM proves superior, these finding would encourage its clinical use.

\section{Disclosure}

Asnis: Speaker's Bureau at Forest Research Institute and Sunovion Pharmaceuticals; Clinical Research Grants from Shire Research, Forest Research Institute, Luudbeck, and Takeda. Henderson reports no conflicts of interest in this work.

\section{References}

1. Rush AJ, Trivedi MH, Wisniewski SR, et al. Acute and longer-term outcomes in depressed outpatients requiring one or several treatment steps: a STAR*D report. Am J Psychiatry. 2006;163(11):1905-1917.

2. Roose SP. Compliance: the impact of adverse events and tolerability on the physician's treatment decisions. Eur Neuropsychopharmacol. 2003;13(Suppl 3):S85-S92.

3. Trivedi MH, Rush AJ, Wisniewski SR et al. Evaluation of outcomes with citalopram for depression using measurement-based care in STAR*D: implications for clinical practice. Am J Psychiatry. 2006;163(1):28-40.

4. Jakubovski E, Bloch MH. Prognostic subgroups for citalopram response in the STAR*D trial. J Clin Psychiatry. 2014;75(7):738-747.
5. American Psychiatric Association. Practice guidelines for the treatment of patients with major depressive disorder (Revision). Am J Psychiatry. 2000;157(Suppl 4):1-45.

6. American Psychiatric Association. Practice guidelines for the treatment of patients with major depressive disorder (Third Edition). Am J Psychiatry. 2010;167(Suppl):1-118.

7. Muramatsu RS, Litzinger MH, Fisher E, Takeshita J. Alternative formulations, delivery methods, and administration options for psychotropic medications in elderly patients with behavioral and psychological symptoms of dementia. Am J Geriatr Pharmacother. 2010;8(2):98-114.

8. Moukaddam NJ, Hirschfeld RM. Intravenous antidepressants: a review. Depress Anxiety. 2004;19(1):1-9.

9. Azzaro AJ, Ziemniak J, Kemper E, Campbell BJ, Vandenberg C. Pharmacokinetics and absolute bioavailability of selegiline following treatment of healthy subjects with the selegiline transdermal system (6 mg/24 h): a comparison with oral selegiline capsules. J Clin Pharmacol. 2007;47(10):1256-1267.

10. Robinson DS, Amsterdam JD. The selegiline transdermal system in major depressive disorder: a systematic review of safety and tolerability. J Affect Disord. 2008;105(1-3):15-23.

11. Bodkin JA, Amsterdam JD. Transdermal selegiline in major depression: a double-blind, placebo-controlled, parallel-group study in outpatients. Am J Psychiatry. 2002;159(11):1869-1875.

12. Amsterdam JD. A double-blind, placebo-controlled trial of the safety and efficacy of selegiline transdermal system without dietary restrictions in patients with major depressive disorder. J Clin Psychiatry. 2003;64(2):208-214.

13. Feiger AD, Rickels K, Rynn MA, Zimbroff DL, Robinson DS. Selegiline trandermal system for the treatment of major depressive disorder: an 8-week, double-blind, placebo-controlled, flexible-dose titration trial. J Clin Psychiatry. 2006;67(9):1354-1361.

14. Amsterdam JD, Bodkin JA. Selegiline transdermal system in the prevention of relapse of major depressive disorder: a 52-week, double-blind, placebo-substitution, parallel-group clinical trial. J Clin Psychopharmacol. 2006;26(6):579-586.

15. Lopez-Munoz F, Alamo C, Juckel G, Assion HJ. Half a century of antidepressant drugs: on the clinical introduction of monoamine oxidase inhibitors, tricyclics, and tetracyclics. Part I: monoamine oxidase inhibitors. J Clin Psychopharmacol. 2007;27(6):555-559.

16. Quitkin FM, Stewart JW, McGrath PJ, et al. Columbia atypical depression. A subgroup of depressives with better response to MAOI than to tricyclic antidepressants or placebo. BrJ Psychiatry Suppl. 1993;(21):30-34.

17. Henkel V, Mergl R, Allgaier AK, Kohnen R, Möller HJ, Hegerl U. Treatment of depression with atypical features: a meta-analytic approach. Psychiatry Res. 2006;141(1):89-101.

18. McGrath PJ, Stewart JW, Harrison W, Wager S, Quitkin FM. Phenelzine treatment of melancholia. J Clin Psychiatry. 1986;47(8):420-422.

19. McGrath PJ, Stewart JW, Janal MN, Petkova E, Quitkin FM, Klein DF. A placebo-controlled study of fluoxetine versus imipramine in the treatment of atypical depression. Am J Psychiatry. 2000;157(3): 344-350.

20. McGrath PJ, Stewart JW, Fava M, et al. Tranylcypromine versus venlafaxine plus mirtazapine following three failed antidepressant medication trials for depression: a STAR*D report. Am J Psychiatry. 2006;163(9):1531-1541.

21. Sunderland T, Cohen RM, Molchan S, et al. High-dose selegiline in treatment-resistant older depressive patients. Arch Gen Psychiatry. 1994;51(8):607-615.

22. Morgan PT. Treatment-resistant depression: response to low-dose transdermal but not oral selegiline. J Clin Psychopharmacol. 2007;27(3): 313-314.

23. Ashton AK. A case report of high-dose transdermal selegiline in the treatment of major depressive disorder. Ann Clin Psychiatry. 2009; 21(1):51-52.

24. Krishnan KR. Revisiting monoamine oxidase inhibitors. J Clin Psychiatry. 2007;68 Suppl 8:35-41. 
25. Ghaemi SN, Hsu DJ, Soldani F, Goodwin FK. Bipolar Disord. Anidepressants in bipolar disorder: the case for caution. 2003;5(6): 421-433.

26. Clary C, Mandos L, Schweitzer E. Results of a brief survey of the prescribing practices for monoamine oxidase inhibitor antidepressants. J Clin Psychiatry. 1990;51(6):226-231.

27. Balon R, Mufti R, Arfken CL. A survey of prescribing practices for monoamine oxidase inhibitors. Psychiatric Services. 1999;50(7):945-947.

28. Shulman KI, Walker SE. Refining the MAOI diet: tyramine content of pizzas and soy products. J Clin Psychiatry. 1999;60(3):191-193.

29. Anderson IM, Ferrier IN, Baldwin RC, et al. Evidence-based guidelines for treating depressive disorders with antidepressants: a revision of the 2000 British Association for Psychopharmacology guideines. J Psychopharmacol. 2008;22(4):343-396.

30. Lam RW, Kennedy SH, Grigoriadis S, et al. Canadian Network for Mood and Anxiety Treatments (CANMAT) clinical guidelines for the management of major depressive disorder in adults, pt 3: Pharmacotherapy. J Affect Disord. 2009;117(Suppl 1):S26-S43.

31. Cascade EF, Kalali AH, Preskorn SH. EMSAM: The first year. Psychiatry (Edgmont). 2007;4(6):19-21.

32. Cooper AJ. Tyramine and irreversible monoamine oxidase inhibitors in clinical practice. Br J Psychiatry. 1989;6(Suppl):38-45.

33. Hendley ED, Snyder SH. The relationship between the action of monoamine oxidase inhibitors on the noradrenaline uptake system and their antidepressant efficacy. Nature. 1968;220(5174):1330-1331.

34. Horwitz D, Lovenberg W, Engelman K, Sjoerdsma A. Monoamine oxidase inhibitors, tyramine, and cheese. JAMA. 1964;(188): $1108-1110$.

35. Asatoor AM, Levi AJ, Milne MD. Tranylcypromine and cheese. Lancet. 1963;2(7310):733.

36. Stahl SM. Stahl's Essential Psychopharmacology: Neuroscientific Basic and Practical Applications. 4th ed. New York: Cambridge University Press; 2013.

37. Pae CU, Bodkin JA, Portland KB, Thase ME, Patkar AA. Safety of selegiline transdermal system in clinical practice: analysis of adverse events from postmarketing exposures. J Clin Psychiatry. 2012;73(5):661-668.

38. Walker SE, Shulmas KI, Tailor SR. Gardner D. Tyramine content of previously restricted foods in monoamine oxidase inhibitor diets. J Clin Psychopharmacol. 1996;16(5):383-388.

39. Sternbach H. The serotonin syndrome. Am J Psychiatry 1991;148(6): 705-713.

40. Kramer M. Sleep loss in resident physicians: the cause of medical errors? Front Neurol. 2010;1(128):1-10.

41. Jacob JE, Wagner ML, Sage JI. Safety of selegiline with cold medications. Ann Pharmacother. 2003;37(3):438-441.

42. Kessler RC, Berglund P, Demler O, et al. The epidemiology of major depressive disorder: results from the National Comorbidity Survey Replication (NCS-R). JAMA. 2003;289(23):3095-3105.

43. Ravindran LN, Stein MB. Pharmacotherapy of post-traumatic stress disorder. Curr Top Behav Neurosci. 2010;(2):505-525.

44. Sinclair LI, Christmas DM, Sean D, et al. Antidepressant-induced jitteriness/anxiety syndrome: systematic review. Br J Psychiatry. 2009; 194(6):483-490

45. Cipriani A, Furukawa TA, Salanti G, et al. Comparative efficacy and acceptability of 12 new-generation antidepressants: a multipletreatments meta analysis. Lancet. 2009;373(9665):746-758.

46. Stahl SM. Enhancing outcomes from major depression: using antidepressant combination therapies with multifunctional pharmacologic mechanisms from the initiation of treatment. CNS Spectr. 2010;15(2):79-94

47. Wimbiscus M, Kostenko O, Malone D. MAO inhibitors: risks, benefits, and lore. Cleve Clin J Med. 2010;77(12):859-882.

48. Chen Y, Kelton CM, Jing Y, Guo JJ, Li X, Patel NC. Utilization, price, and spending trends for antidepressants in the US Medicaid program. Res Social Adm Pharm. 2008;4(3):244-257.
49. Soutullo C, Figueroa-Quintana A. When do you prescribe antidepressants to depressed children? Curr Psychiatry Rep. 2013; 15(7):366.

50. Meyers BS, Jeste DV. Geriatric psychopharmacology: evolution of a discipline. J Clin Psychiatry. 2010;71(11):1416-1424.

51. Bridge JA, Birmaher B, Iyengar S, Barbe RP, Brent DA. Placebo response in randomized controlled trials of antidepressants for pediatric major depressive disorder. Am J Psychiatry. 2009;166(1):42-49.

52. Reynolds CF 3rd, Frank E, Kupfer DJ, et al. Treatment outcome in recurrent major depression: A post hoc comparison of elderly ("young old") and midlife patients. Am J Psychiatry. 1996;153(10):1288-1292.

53. Cheung AH, Emslie GJ, Mayes TL. The use of antidepressants to treat depression in children and adolescents. CMAJ. 2006;174(2):193-200.

54. Slotkin TA, Seidler FJ, Ritchie JC. Regional differences in brain monoamine oxidase subtypes in an animal model of geriatric depression: effects of olfactory bulbectomy in young versus aged rats. Brain Res. 2000;882(1-2):149-154.

55. Meyer JH, Ginovart N, Boovariwala A, et al. Elevated monoamine oxidase a levels in the brain: an explanation for the monoamine imbalance of major depression. Arch Gen Psychiatry. 2006;63(11):1209-1216.

56. Slotkin TA, Whitmore WL, Barnes GA, et al. Reduced inhibitory effect of imipramine on radiolabeled serotonin uptake into platelets in geriatric depression. Biol Psychiatry. 1989;25(6):687-691.

57. DelBello MP, Hochadel TJ, Portland KB, et al. A double-blind, placebocontrolled study of selegiline transdermal system in depressed adolescents. J Child Adolesc Psychopharmacol. 2014;24(6):311-317.

58. Kupfer DJ. The pharmacological management of depression. Dialogues Clin Neurosci. 2005;7(3):191-205.

59. Hansen DL, Tulinius D, Hansen EH. Adolescents' struggles with swallowing tablets: barriers, strategies and learning. Pharm World Sci. 2008;30(1):65-69.

60. Wright D. Medication administration in nursing homes. Nurs Stand. 2002;16(42):33-38.

61. Asberg M. Treatment of depression with tricyclic drugs-pharmacokinetic and pharmacodynamic aspects. Pharmakopsychiatr Neuropsychopharmakol. 1976;9(1):18-26.

62. Asnis GM, Friedman TA, Sanderson WC, Kaplan ML, van Praag HM, Harkavy-Friedman JM. Suicidal behaviors in adult psychiatric outpatients, I: Description and prevalence. Am J Psychiatry. 1993;150(1):108-112.

63. EMSAM [package insert]. Napa, CA: Dey Pharma; 2009.

64. Wiesbrock UO, Rocholz G, Franzelius C, Schwark T, Grellner W. Excessive use of fentanyl patches as the only means of suicide. Arch Kriminol. 2008;222(1-2):23-30.

65. Lovecchio F, Ramos L. Suicide by Duragesic transdermal fentanyl patch toxicity. Am J Emerg Med. 2010;29(1):131.

66. Raber JH, Shinar C, Finkelstein S. Clonidine patch ingestion in an adult. Ann Pharmacother. 1993;27(6):719-722.

67. Woolf A, Burkhart K, Caraccio T, Litovitz T. Self-poisoning among adults using multiple transdermal nicotine patches. J Toxicol Clin Toxicol. 1996;34(6):691-698.

68. Angst J, Azorin JM, Bowden CL, et al. Prevalence and characteristics of undiagnosed bipolar disorders in patients with a major depressive episode: the BRIDGE study. Arch Gen Psychiatry. 2011;68(8):791-798.

69. Tondo L, Vazquez G, Baldessarini RJ. Mania associated with antidepressant treatment: comprehensive meta-analytic review. Acta Psychiatr Scand. 2010;121(6):404-414.

70. Fava M, Rush AJ, Alpert JE, et al. Difference in treatment outcome in outpatients with anxious versus nonanxious depression: a STAR*D report. Am J Psychiatry. 2008;165(3):342-351.

71. Papakostas GI, Stahl SM, Krishen A, et al. Efficacy of bupropion and the selective serotonin reuptake inhibitors in the treatment of major depressive disorder with high levels of anxiety (anxious depression): a pooled analysis of 10 studies. J Clin Psychiatry. 2008;69(8);1287-1292.

72. Klein DF, Liebowitz MR. Hysteroid dysphoria. Am J Psychiatry. 1982;139(11):1520-1521. 
73. Robinson D, Portland KB. Selegiline transdermal system (STS) for anxious depression: A post hoc analysis of 3 randomized, placebocontrolled, double-blind studies. Presented at the Annual Meeting of the American Psychiatric Association; May 15, 2011; Honolulu, Hawaii.

74. Asnis GM, McGinn LK, Sanderson WC. Atypical depression: clinical aspects and noradrenergic function. Am J Psychiatry. 1995;152(1): $31-36$.
75. Stewart JW, McGrath PJ, Fava M, et al. Do atypical features affect outcome in depressed outpatients treated with citalopram? Int J Neuropsychopharmacol. 2010;13(1):15-30.

76. Pae CU, Patkar AA, Jang S, Portland KB, Jung S, Nelson JC. Efficacy and safety of selegiline transdermal system (STS) for the atypical subtype of major depressive disorder: pooled analysis of 5 short-term, placebo-controlled trials. CNS Spectr. 2013;(29):1-6.

\section{Publish your work in this journal}

Neuropsychiatric Disease and Treatment is an international, peerreviewed journal of clinical therapeutics and pharmacology focusing on concise rapid reporting of clinical or pre-clinical studies on a range of neuropsychiatric and neurological disorders. This journal is indexed on PubMed Central, the 'PsycINFO' database and CAS, and is the official journal of The International Neuropsychiatric Association (INA). The manuscript management system is completely online and includes a very quick and fair peer-review system, which is all easy to use. Visit http://www.dovepress.com/testimonials.php to read real quotes from published authors.

Submit your manuscript here: http://www.dovepress.com/neuropsychiatric-disease-and-treatment-journal 